\title{
Impacto de la pandemia de COVID-19 y la cuarentena en hombres que tienen sexo con hombres viviendo con VIH en Argentina
}

\author{
Recibido: 30/10/20 Aceptado: 16/11/20
}

Jamile Ballivian ${ }^{1}$, Diego Cecchini', María Luisa Alcaide ${ }^{2}$, John Abbamonte ${ }^{3}$, Deborah Jones ${ }^{3}$ e Isabel Cassetti ${ }^{1}$.

\section{RESUMEN}

Introducción: Las personas que viven con VIH (PVV) tienen mayor riesgo de complicaciones de salud mental. Para mitigar la propagación del COVID-19 en nuestro país se estableció una cuarentena temprana. Estudiamos el impacto del estrés relacionado con COVID-19 y la cuarentena en hombres que tienen sexo con hombres que viven con $\mathrm{VIH}$ (HSHV) en Argentina.

Materiales y métodos: Realizamos una encuesta anónima en abril-mayo de 2020. Se incluyeron PVV en seguimiento en una institución privada en Argentina. Se evaluaron la adherencia a medidas preventivas de COVID-19 y al tratamiento antirretroviral, el acceso a recursos, factores psicosociales y el consumo de sustancias durante la cuarentena. Este análisis se centró en los resultados de HSHV.

Resultados: Entre 1336 participantes incluidos, 596 se autoreportaron HSHV (mediana de edad de 44 años, RIC 3552). La adherencia a la cuarentena fue del $96,8 \%$; la pérdida de empleo en el hogar $55,4 \%$. Se observó interrupción en la obtención de medicamentos antirretrovirales en el 4,9\% y el 29,9\% reportó adherencia subóptima. Los problemas de salud mental fueron frecuentes: depresión (14,1\%), soledad $(15,6 \%)$ y ansiedad $(20,2 \%)$.

Conclusiones: Nuestro estudio evidenció alta adherencia a las medidas preventivas al inicio de la cuarentena y dificultades en el acceso a los servicios de salud. La pandemia de COVID-19 ha comprometido la economía del país; este estudio muestra su impacto en el acceso de los

\begin{abstract}
${ }^{1}$ Helios Salud, Buenos Aires, Argentina.
${ }^{2}$ University of Miami Miller School of Medicine, Department of Medicine, Miami, USA.

${ }^{3}$ University of Miami Miller School of Medicine, Department of Psychiatry \& Behavioral Sciences, Miami, USA.

Dirección para la correspondencia: Dra. Jamile Ballivian, Helios Salud SA. Perú 1511/15, Buenos Aires, C1141ACG, Argentina. Tel: +5411 43637400 . Email: jballivian@heliossalud.com.ar

Los autores no presentan conflictos de interés en relación con el manuscrito. El trabajo fue presentado en parte en HIV Glasgow Drug Therapy Virtual Congress, 5-8 octubre 2020, póster \#132.
\end{abstract}

HSHV a recursos básicos. Dado que la salud mental de los HSHV ha sido afectada, la pandemia resalta la necesidad de una visión integral de la salud de nuestros pacientes.

Palabras clave: VIH, COVID-19, Argentina, salud mental. 


\section{Introducción}

En diciembre de 2019 se reportaron los primeros casos de neumonía de origen desconocido en Wuhan, China ( 1 , 2). El estudio de este brote permitió a principios de 2020 la identificación de un nuevo betacoronavirus (3), que fue denominado coronavirus del síndrome respiratorio agudo grave 2 (SARS-CoV-2) y definido como el agente causal de la enfermedad por coronavirus 2019 (COVID-19) (4). En enero de 2020, la Organización Mundial de la Salud (OMS) declaró el brote mundial de COVID-19 como una emergencia de salud pública (5) y, posteriormente, tras comprometer los seis continentes, la pandemia a partir del 11 de marzo de $2020(6,7)$.

El crecimiento exponencial en el número de casos a nivel mundial ha llevado a implementar estrategias para restringir el movimiento de la población (cuarentena masiva) con el objetivo de controlar la epidemia y evitar la saturación de los sistemas de atención de salud. Estas restricciones se han aplicado en numerosos países y se han desarrollado protocolos regionales que incluyen restricciones a las reuniones sociales, recomendaciones para el distanciamiento social, cierre de escuelas, restricciones en el transporte (a nivel jurisdiccional, nacional e internacional), y limitación de toda actividad no esencial $(8,9)$.

Sehaestablecido recientementeque las medidas de cuarentena podrían tener efectos psicológicos negativos, generando síntomas de estrés postraumático, estrés, ansiedad y depresión (8). Más allá del impacto psicológico directo del aislamiento en los individuos, la cuarentena trae como consecuencia la pérdida de acceso a servicios, reducción de los ingresos o riesgo de desempleo y aislamiento social que pueden afectar considerablemente la salud mental de la población. El estrés afecta negativamente los comportamientos de salud y está asociado con deterioro de la salud física y emocional. Esto puede tener impacto en poblaciones especiales como aquellas de bajos recursos y con patologías crónicas, como las personas que viven por VIH/SIDA (PVV) $(8,10-13)$.

Argentina es, al momento de redacción de este artículo, uno de los países más afectados por la pandemia de COVID-19 a nivel mundial (7). Si bien el primer caso fue reportado el 3 de marzo de 2020 (14), el Estado Nacional decretó el inicio del Aislamiento Social Preventivo y Obligatorio (ASPO) (15) el 20 de marzo del corriente año. A pesar de las medidas precozmente instauradas, el número de infecciones se ha incrementado sostenidamente desde entonces. Al 25 de octubre de 2020 se han detectado 1.090 .589 casos y 28.896 fallecidos por COVID-19 en nuestro país (16). Si bien al inicio la epidemia presentó su epicentro en el Área Metropolitana de Buenos Aires, actualmente se ha expandido a todo el país (14).

Según datos reportados por el Ministerio de Salud de Argentina, se estima que en el país viven 139 mil personas viven con $\mathrm{VIH}$, de las cuales solamente el $80 \%$ conoce su diagnóstico. La relación varón/mujer es de 2.5 a 1, siendo la principal vía de transmisión las relaciones sexuales sin uso de preservativo (98\%). Entre los varones, la mitad de las infecciones se producen durante relaciones sexuales con mujeres y la otra mitad durante prácticas sexuales con otros varones (17). Un estudio reciente en personas adultas con VIH/SIDA evidenció que la pandemia de COVID-19 y la cuarentena tienen impacto en la salud mental de esta población. Se detectaron proporciones de estrés del $35 \%$, ansiedad $20 \%$ y depresión $16 \%$, entre otras variables (18).

La pandemia de COVID-19 ha incrementado las inequidades en salud entre las poblaciones vulnerables a nivel mundial, entre ellas el colectivo de hombres que tienen sexo con hombres (HSH) (13). Dentro de las PVV, la población de hombres que tienen sexo con hombres que viven con $\mathrm{VIH}$ (HSHV) constituye un grupo particularmente vulnerable a eventos adversos en el contexto de la pandemia de COVID-19. Esta población presenta riesgo de desempleo, de vulneración de su privacidad en el contexto de estrategias de seguimiento y mitigación de casos de COVID-19, de discontinuación del tratamiento antirretroviral y del acceso al sistema de salud y de desarrollo de trastornos de salud mental $(13,19-21)$.

Se ha descripto que la prevalencia de trastornos mentales (alteraciones del estado de ánimo, ansiedad y abuso de sustancias) es mayor entre hombres que tienen sexo con hombres $(\mathrm{HSH})$ que entre hombres heterosexuales (12, $20,22)$. Se estima que las tasas de depresión y estrés son un $17 \%$ más altas en los HSH en comparación con los hombres heterosexuales, así como también son mayores las prevalencias de trastorno de estrés postraumático y consumo de sustancias. En este contexto, existe una imperiosa necesidad de definir el impacto de la pandemia de COVID-19 y la cuarentena en la salud mental, aspectos económicos y adherencia al tratamiento antirretroviral de la población de HSHV en Argentina.

\section{Materiales y métodos}

Tras la aprobación del comité de ética en investigación (CEI) se llevó a cabo una encuesta anónima a PVV mayores de 18 años de forma electrónica en la plataforma Redcap entre abrily mayo de 2020. Este estudio se realizó entre sujetos asistidos en Helios Salud, una clínica ambulatoria especializada en el tratamiento 
de $\mathrm{VIH}$, que cuenta con una red de prestadores en todo el país. Esta institución se caracteriza por brindar un seguimiento integral de las PVV proveyendo testeo, seguimiento clínico, de laboratorio, soporte psicosocial, dispensación de medicación y teleconsulta implementada en contexto de la pandemia.

Los candidatos fueron identificados en la base de datos de todo el país de la institución. Se contactó a aquellos que previamente consintieron para ser contactados por el centro electrónicamente y que se encontraban en seguimiento activo. Dada la naturaleza del estudio, una encuesta anónima administrada en línea, el CEI no requirió solicitud de consentimiento informado por escrito y el mismo se obtuvo por vía electrónica al contactar a los participantes por WhatsApp o correo electrónico y antes de completar la encuesta.

\section{Instrumento de recolección}

Se administró una forma adaptada de la encuesta COVID-19 del estudio combinado de cohortes MACS/ WIHS (MWCCS) (23). La encuesta incluyó variables sociodemográficas (edad, género), factores de riesgo de COVID-19 y adherencia al régimen de tratamiento antirretroviral, antecedentes de síntomas y pruebas COVID actuales, uso de tabaco y marihuana, cambios de recursos, violencia y factores psicosociales, afrontamiento, salud mental/depresión, estrés percibido y soledad. El detalle de los instrumentos de medición y su adaptación para nuestra población fue publicado previamente (18). La satisfacción con el apoyo social se midió utilizando el ítem "En el último mes, ¿qué tan satisfecho está con el apoyo social que ha recibido de otros?", otorgando el paciente un puntaje en una escala tipo Likert de 5 puntos. Las dificultades económicas se evaluaron utilizando un ítem que preguntó si el paciente presentó "Dificultad para pagar las necesidades básicas, incluida la comida y ropa", caracterizándose como variable dicotómica (SÍ/NO). Para este análisis la frecuencia de depresión autopercibida se midió con una escala tipo Likert de 4 puntos $(0=$ nunca y 4=siempre) utilizando el ítem "Me sentí deprimido". La soledad se midió utilizando una forma adaptada del Formulario Breve de Soledad (Loneliness Brief Form). Esta medida aplica una escala tipo Likert de 5 puntos. La ansiedad autopercibida se midió con una escala tipo Likert de 4 puntos ( $0=$ nunca y $4=$ siempre), utilizando el ítem "Me sentí nerviosa/o". El uso de drogas se calificó con un elemento que preguntaba: "¿Ha consumido drogas durante la cuarentena?" Sí/NO (dicotómico). En caso de responder "Sí" se debía indicar cuál o cuáles. Por último, la violencia doméstica se evaluó mediante dos preguntas,

\section{Figura 1. Participantes}

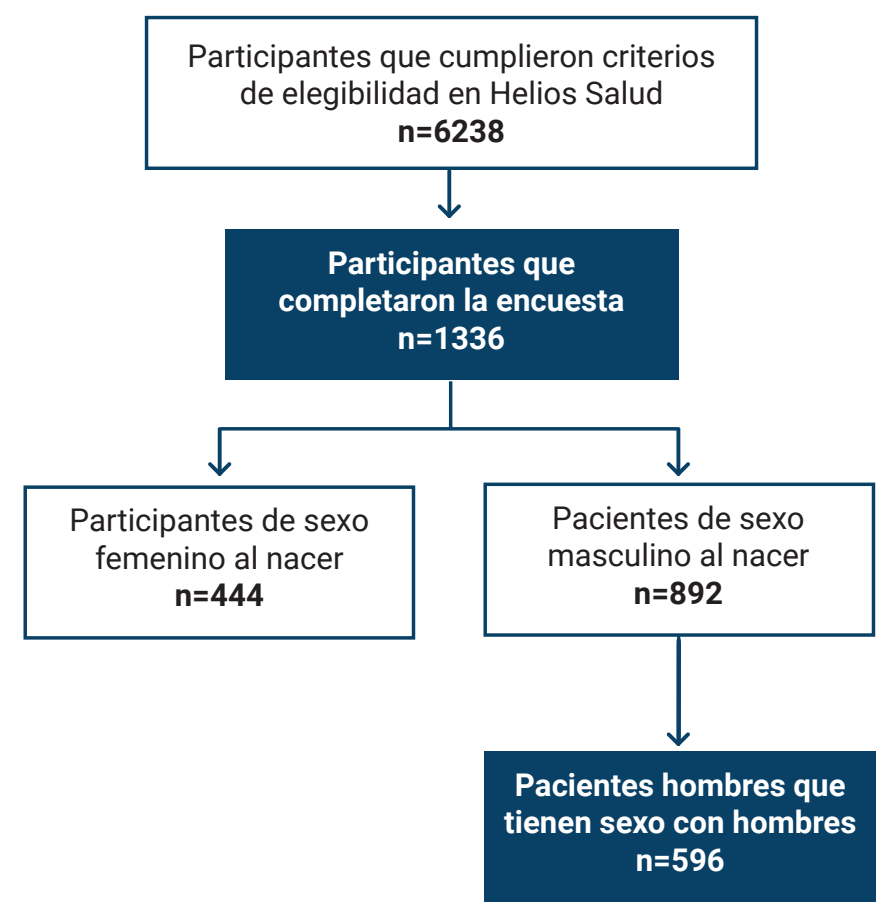

la primera consultando si experimentó violencia física, abuso sexual o emocional y una segunda indagando si experimentó un incremento en alguna de las anteriores.

Las características epidemiológicas e inmunovirológicas de la población contactada se obtuvieron del análisis de las bases de datos correspondientes a la historia clínica electrónica institucional (software InfHos). Para este manuscrito se realizó un subestudio de la población de HSHV que participó en la encuesta mencionada. Se realizó un análisis descriptivo de las variables evaluadas utilizando el software STATA 13.

\section{Resultados}

En la red de centros de Helios Salud se encontraban 10.300 pacientes en seguimiento al momento de la realización de este estudio. Del total de pacientes, fueron elegibles 6238 sujetos y pudieron ser contactados por medios electrónicos 5018 (Figura 1). Las características inmunovirológicas de la población elegible fueron publicadas previamente(18);Tabla 1.

La mediana (rango intercuartílico, IQR) de linfocitos T-CD4+ fue de 626 células/mL (442-823), el 95,2\% ( $n=4777)$ presentó carga viral $<200$ copias $/ m L$ y el $98,7 \%(n=98,7 \%)$ se encontraba bajo tratamiento antirretroviral. Ingresaron a la encuesta 2126 pacientes (42,3\%); de los cuales 1336 aceptaron participar y completaron la encuesta; 892 hombres y 444 mujeres. El rango de edad de los participantes fue entre 18-82 años, siendo 


\section{Tabla 1. Características de los pacientes elegibles a los que se envió la encuesta}

\begin{tabular}{|c|c|}
\hline Variables & $N=5018$ \\
\hline Edad, media (DS) & $45.1(11.0)$ \\
\hline Último recuento de CD4+, (IQR) & $626(442-823)$ \\
\hline 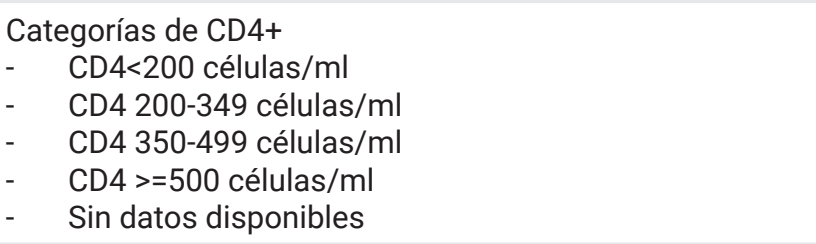 & $\begin{array}{c}190(3.8 \%) \\
540(10.8 \%) \\
901(18.0 \%) \\
3357(66.9 \%) \\
30(0.6 \%)\end{array}$ \\
\hline $\begin{array}{l}\text { Copias de ARN HIV-1 (carga viral) } \\
-\quad<200 \text { copias/ml, no detectable } \\
-\quad>=200 \text { copias/ml, detectable } \\
\text { - } \quad \text { Sin datos disponibles }\end{array}$ & $\begin{array}{c}4777(95.2 \%) \\
219(4.36 \%) \\
22(0.44 \%)\end{array}$ \\
\hline $\begin{array}{l}\text { Tratamiento antiretroviral } \\
-\quad \mathrm{No} \\
-\quad \mathrm{Si}\end{array}$ & $\begin{array}{c}64(1.3 \%) \\
4954(98.7 \%)\end{array}$ \\
\hline
\end{tabular}

Tabla modificada de Ballivian J, Alcaide ML, Cecchini D, Jones DL, Abbamonte JM, Cassetti I. Impact of CoVID-19-related stress and lockdown on mental health among people living with HIV in Argentina. J Acquir Immune Defic Syndr. 2020 Sep 3

residentes en su mayoría en el Área Metropolitana de Buenos Aires $(94,1 \%, n=1257)$ y correspondiendo el porcentaje restante a PVV del interior del país.

De los participantes de sexo masculino, 596 (66\%) se autoreportaron como HSHV, con una mediana de edad de 44 años (IQR 35-52), tal como se muestra en la Tabla 2.

\section{Impacto económico de la cuarentena}

Esta población presentó una alta adherencia a la cuarentena $(96,8 \% \mathrm{n}=577)$. Con respecto a los factores de impacto económico, se observó una elevada proporción de individuos que reportaron dificultades para obtener necesidades básicas como alimentos o ropa $36,6 \%(n=218)$. A nivel laboral, el $55,4 \%$ $(n=330)$ de los sujetos reportaron disminución de las horas de trabajo, interrupción del empleo o pérdida de empleo en su hogar. Dentro de este subgrupo, $27,3 \%$ solicitó o recibió subsidios estatales ( $n=90 / 330$ ).

\section{Acceso al sistema de salud y adherencia a medicación}

En cuanto a la vinculación con el sistema de salud, 27,2\% $(\mathrm{n}=162)$ informó problemas para acceder a los servicios médicos proporcionados por telemedicina. La interrupción en la obtención de medicamentos no antirretrovirales ocurrió en $7,7 \%(n=46)$ de los sujetos y $4,9 \%(n=29)$ en el caso de medicación antirretroviral.

Con respecto a la adherencia a la medicación antiretroviral, el $70,1 \%(n=418)$ reportó adherencia excelente; $20,1 \%$ muy buena $(n=120) ; 6,7 \%$ buena $(n=40) ; 1,7 \%$ regular $(n=10)$; $0,5 \%$ mala $(n=3)$ y $0,8 \%$ muy mala $(n=5)$.

\section{Salud mental}

En esta población los problemas de salud mental autopercibidos fueron frecuentes: depresión $(14,1 \% ; n=84)$, soledad $(15,6 \%$; $n=93)$ y ansiedad $(20,2 \% ; n=120)$. El 6,5\% ( $n=39)$ informó haber presentado alguna forma de abuso (sexual, emocional o físico); y $6 \%(n=36)$ reportó un incremento del abuso emocional durante la cuarentena. El 13,1\% $(n=78)$ refirió un aumento en el consumo de alcohol en el mismo período.

\section{Discusión}

La pandemia de COVID-19 ha impactado drásticamente en la salud pública a nivel mundial, representando un desafío sin precedentes para la salud pública en Argentina. Por este motivo, los efectos de la pandemia requieren un abordaje interdisciplinario que amplíe el enfoque más allá de los indicadores epidemiológicos (8) y de los aspectos biológicos de la enfermedad. Nuestro trabajo refleja el impacto inicial de la pandemia y el aislamiento en la población de HSHV, un colectivo cuya vulnerabilidad ha sido establecida previamente.

A pesar de su extendida implementación como medida de contención de la epidemia, diferentes autores han planteado el impacto negativo de las medidas de aislamiento en la salud mental de la población general. La extensión de este fenómeno ha llevado a hipotetizar que este efecto deletéreo podría incluso devenir en una pandemia de trastornos de salud mental (11) 


\section{Tabla 2. Demografía, tratamiento y factores psicosociales de los participantes hombres que} tienen sexo con hombres $(n=596)$

\begin{tabular}{|c|c|}
\hline Variable & n (\%) / Mediana (IQR) \\
\hline Edad & $44(35.3-52.3)$ \\
\hline Sexo al nacer masculino & $596(100 \%)$ \\
\hline $\begin{array}{l}\text { Incapacidad de obtener atención por telemedicina } \\
-\quad \text { No } \\
-\quad \text { Sí }\end{array}$ & $\begin{array}{l}434(72.8 \%) \\
162(27.2 \%)\end{array}$ \\
\hline $\begin{array}{l}\text { Problemas para obtener medicamentos contra el VIH } \\
-\quad \text { No } \\
-\quad \text { Sí }\end{array}$ & $\begin{array}{l}434(72.8 \%) \\
162(27.2 \%)\end{array}$ \\
\hline $\begin{array}{l}\text { Problemas para obtener otros medicamentos } \\
-\quad \text { No } \\
-\quad \text { Sí }\end{array}$ & $\begin{array}{c}550(92.3 \%) \\
46(7.7 \%)\end{array}$ \\
\hline $\begin{array}{ll}\text { Capacidad para adherir a la medicación para el VIH } \\
- & \text { Muy malo } \\
- & \text { Malo } \\
- & \text { Regular } \\
- & \text { Buena } \\
- & \text { Muy buena } \\
- & \text { Excelente }\end{array}$ & $\begin{array}{c}5(0.8 \%) \\
3(0.5 \%) \\
10(1.7 \%) \\
40(6.7 \%) \\
120(20.1 \%) \\
418(70.1 \%)\end{array}$ \\
\hline $\begin{array}{l}\text { Interrupción del cuidado de la salud mental } \\
-\quad \text { No recibe atención de salud mental } \\
-\quad \text { De nada } \\
-\quad \text { Un poco } \\
\text { - }\end{array}$ & $\begin{array}{l}502(84.2 \%) \\
30(5 \%) \\
30(5 \%) \\
34(5.7 \%)\end{array}$ \\
\hline $\begin{array}{l}\text { Interrupción de la atención por abuso de sustancias } \\
-\quad \text { No recibe atención de abuso de sustancias } \\
-\quad \text { Sin interrupción } \\
-\quad \text { Algo } \\
\text { - } \quad \text { Mucho }\end{array}$ & $\begin{array}{l}588(98.7 \%) \\
3(0.5 \%) \\
1(0.2 \%) \\
4(0.7 \%)\end{array}$ \\
\hline $\begin{array}{l}\text { Antecedente de abuso (físico, emocional, sexual) } \\
-\quad \text { No } \\
-\quad \text { Sí }\end{array}$ & $\begin{array}{c}557(93.5 \%) \\
39(6.5 \%)\end{array}$ \\
\hline $\begin{array}{l}\text { Aumento del abuso durante la cuarentena (físico) } \\
-\quad \text { No } \\
-\quad \text { Sí }\end{array}$ & $\begin{array}{c}590(99 \%) \\
6(1 \%)\end{array}$ \\
\hline $\begin{array}{l}\text { Aumento del abuso durante la cuarentena (emocional) } \\
-\quad \text { No } \\
-\quad \text { Sí }\end{array}$ & $\begin{array}{c}560(94 \%) \\
36(6 \%)\end{array}$ \\
\hline $\begin{array}{l}\text { Aumento del abuso durante la cuarentena (sexual) } \\
-\quad \text { No } \\
-\quad \text { Sí }\end{array}$ & $\begin{array}{l}590(99 \%) \\
6(1 \%)\end{array}$ \\
\hline Depresión & $84(14.1 \%)$ \\
\hline Soledad & $93(15,6 \%)$ \\
\hline Ansiedad & $120(20,2 \%)$ \\
\hline $\begin{array}{l}\text { Dificultades económicas para pagar necesidades básicas incluyendo ropa y comida } \\
-\quad \text { No } \\
-\quad \text { Sí }\end{array}$ & $\begin{array}{l}378(63.4 \%) \\
218(36.6 \%)\end{array}$ \\
\hline $\begin{array}{l}\text { Disminución de horas de trabajo, interrupción del empleo o pérdida de empleo en el hogar } \\
-\quad \mathrm{No} \\
-\mathrm{Si}\end{array}$ & $\begin{array}{l}266(44.6 \%) \\
330(55.4 \%)\end{array}$ \\
\hline $\begin{array}{l}\text { Requerimiento o recepción de subsidios estatales por desempleo n=330/596 } \\
-\quad \text { No } \\
-\quad \text { Sí }\end{array}$ & $\begin{array}{c}240(72.7 \%) \\
90(27.3 \%)\end{array}$ \\
\hline $\begin{array}{l}\text { Consumo de drogas durante la cuarentena } \\
-\quad \text { No } \\
-\quad \text { Sí }\end{array}$ & $\begin{array}{l}428(71.8 \%) \\
168(28.2 \%)\end{array}$ \\
\hline
\end{tabular}

*Nota. Variables categóricas notificadas como n (\%) y variables continuas como mediana (IQR) 
debido al mayor el riesgo de soledad, aislamiento y ansiedad. A nivel local, la cuarentena y la crisis económica en nuestro país profundizaron la dificultad de acceso al trabajo, el riesgo de desempleo, la inestabilidad en la vivienda y la inseguridad alimentaria.

Se ha descripto en la literatura que la frecuencia relativamente alta de problemas de salud mental entre los $\mathrm{HSH}$ es aun mayor entre los HSHV. En un estudio de más de 500 HSHV, el $47 \%$ cumplió con los criterios de diagnóstico para un trastorno de ansiedad, $22 \%$ para trastornos depresivos y $25 \%$ con síntomas compatibles con abuso de sustancias (24). Estas comorbilidades pueden tener un efecto deletéreo en la adherencia al tratamiento antirretroviral, exponiendo a los individuos al riesgo de fallo virológico con el consecuente riesgo de deterioro inmunológico y clínico.

En este contexto, nuestra investigación constituye el primer subanálisis en población de HSHV en Argentina que contempla la adherencia a medidas de cuarentena de COVID-19, impacto económico, psicosocial, acceso a recursos sanitarios y adherencia al tratamiento antirretroviral.

Con respecto a las medidas de prevención de COVID-19, se ha observado una notable adherencia a las medidas de restricción de circulación en esta población. Sin embargo, como ocurrió con la población general, esto pudo haber repercutido negativamente en el acceso al sistema de salud, especialmente para aquellos que, ante la imposibilidad de concurrir a visitas presenciales, no pudieron acceder a consultas por telemedicina. A nivel mundial, se ha observado un incremento del 80 al $100 \%$ de la disponibilidad de consultas por telemedicina para mitigar el impacto sanitario de la pandemia (25-27). A nivel local, no se cuenta con estadísticas oficiales sobre esta nueva modalidad de atención. Dado que en Helios Salud la telemedicina no era una práctica habitual hasta el advenimiento de la pandemia, las dificultades específicas en el acceso a este recurso no fueron evaluadas en esta investigación. A priori hipotetizamos que tanto las limitaciones en el acceso a los recursos tecnológicos, así como la falta de costumbre de los pacientes a utilizar este medio, pudieron ser factores determinantes en el acceso a esta prestación. Análisis posteriores a la fase de implementación de telemedicina en la institución demostraron que la adherencia a telemedicina es alta, mayor al 85\% (datos no publicados).

El impacto económico y la dificultad en el acceso a necesidades básicas en este colectivo también fue evidenciado por nuestro estudio. Más de la mitad de los HSHV encuestados presentaron riesgo o pérdida de empleo en su hogar. Una encuesta recientemente realizada durante la pandemia de
COVID-19 en Estados Unidos describió un incremento de un tercio en la inseguridad alimentaria en la población estudiada, tendiendo esto a correlacionarse con la pérdida de empleo (28). En nuestro estudio, la alta proporción de dificultad en relación al empleo podría vincularse con la elevada dificultad en el acceso a necesidades básicas reportado por esta población.

Con respecto a parámetros de salud mental, esta encuesta provee datos sobre la población HSHV que reflejan el impacto precoz de la pandemia y la cuarentena. Al momento de la escritura de estemanuscrito (octubre 2020) nuestro país llevaba más de 200 días con diferentes niveles de aislamiento. Se ha descrito en la literatura que cuarentenas mayores a diez días tienen un mayor impacto emocional (estrés postraumático) (8). A pesar de haber sido un estudio realizado en los inicios del aislamiento en nuestro país, el mismo refleja el impacto del ASPO con más de diez días de duración, momento en el que la restricción a la circulación era más estricta y se limitaba solo a actividades esenciales. En este contexto, esta investigación provee datos basales que podrán ser comparados con estudios subsiguientes realizados tras la prolongación y los cambios instaurados en el ASPO en diferentes regiones.

Nuestro estudio presenta varias limitaciones que deben considerarse y que disminuyen su validez externa. En primer lugar, se trata de pacientes asistidos bajo el sistema de obras sociales, que forman parte del sistema de empleo formal. Dicha población difiere de la que se atiende en el subsistema de salud público, ya que el acceso al empleo podría proveerle mejores herramientas para afrontar situaciones de estrés psíquico y socioeconómico. Cabe destacar que en nuestro país el $63 \%$ de las PVV es asistida por el sistema de salud público (17), por lo que es necesario un diagnóstico de situación en este grupo. Por otra parte, nuestra institución fortaleció sus estándares de atención disponibles: envío a domicilio de la medicación monitoreo remoto de adherencia, así como la provisión por más de un mes en pacientes con tratamiento estable. Inferimos que lo antedicho contribuyó al bajo porcentaje de pacientes con dificultades de acceso al tratamiento antirretroviral reportado. Del mismo modo, la institución implementó una estrategia de telemedicina innovadora basada en la aplicación WhatsApp que se puso en funcionamiento en menos de dos semanas. Por todo lo expuesto, consideramos que la posibilidad de extrapolación de estos resultados a HSHV que se atienden en el subsector público es limitada.

En segundo lugar, este estudio, dado su enfoque predominantemente psicosocial, no ha evaluado específicamente parámetros virológicos ni inmunológicos de los participantes que han respondido la encuesta, sino que 
a los fines de esta descripción se tomaron datos generales disponibles en la base de datos institucional de la población contactada. Por este motivo, el impacto de la pandemia y la cuarentena en la carga viral y los CD4 de esta población de HSHV deberá ser objeto de futuros estudios que evalúen estas variables específicamente.

Las prevalencias observadas de depresión y ansiedad autopercibidas en la población de HSHV en nuestro estudio deben tenerse en consideración en el abordaje integral de esta población frente a esta contingencia. Es necesario desarrollar estrategias que contemplen la salud integral de los PVV, tanto mental tanto como física, dado que estos parámetros pueden tener incidencia en la adherencia al tratamiento antirretroviral. Nuestra investigación puso en evidencia que, pese a que los participantes tuvieron una baja prevalencia de dificultades en el acceso a la medicación, la proporción de adherencia subóptima (menos que excelente) fue mayor. En este contexto, es necesario implementar medidas que permitan garantizar tanto el acceso a la medicación como apoyo psicosocial para facilitar estrategias de afrontamiento que permitan tanto mejorar como evitar deterioros en la adherencia a la misma.

En conclusión, nuestro estudio evidencia que el ASPO, a pesar de haber contribuido al fortalecimiento del sistema de salud y contención inicial de la pandemia, ha tenido impacto en aspectos psicosociales y en el acceso al sistema de salud de la población de HSHV. Se requieren estudios que incluyan a PVV en seguimiento por el subsector público y con mayor participación del interior del país a fin de realizar un diagnóstico de situación más amplio en esta población. Esta información permitirá desarrollar y fortalecer las políticas públicas que provean un enfoque integral de la salud física y psicosocial de las PVV. 


\section{Referencias}

1. Guan W-J, Ni Z-Y, Hu Y, Liang W-H, Ou C-Q, He J-X, et al. Clinical Characteristics of Coronavirus Disease 2019 in China. N Engl J Med. 2020 Apr;382(18):1708-20.

2. Li Q, Guan X, Wu P, Wang X, Zhou L, Tong Y, et al. Early Transmission Dynamics in Wuhan, China, of Novel Coronavirus-Infected Pneumonia. N Engl J Med. 2020 Mar;382(13):1199-207.

3. Lu R, Zhao X, Li J, Niu P, Yang B, Wu H, et al. Genomic characterisation and epidemiology of 2019 novel coronavirus: implications for virus origins and receptor binding. Lancet (London, England). 2020 Feb;395(10224):565-74.

4. Ludwig S, Zarbock A. Coronaviruses and SARS-CoV-2: A Brief Overview. Anesth Analg. 2020 Jul;131(1):936.

5. Wathelet M, Duhem S, Vaiva G, Baubet T, Habran E, Veerapa E, et al. Factors Associated With Mental Health Disorders Among University Students in France Confined During the COVID-19 Pandemic. JAMA Netw open. 2020 Oct;3(10):e2025591.

6. World Health Organization. Coronavirus disease (COVID-19) Situation Report - 200. 2020.

7. World Health Organization. WHO coronavirus disease (COVID-19) dashboard. Geneva; 2020.

8. Brooks SK, Webster RK, Smith LE, Woodland L, Wessely S, Greenberg N, et al. The psychological impact of quarantine and how to reduce it: rapid review of the evidence. Lancet (London, England). 2020 Mar;395(10227):912-20.

9. Di Gennaro F, Pizzol D, Marotta C, Antunes M, Racalbuto V, Veronese N, et al. Coronavirus Diseases (COVID-19) Current Status and Future Perspectives: A Narrative Review. Int J Environ Res Public Health. 2020 Apr;17(8).

10. Talevi D, Socci V, Carai M, Carnaghi G, Faleri S, Trebbi E, et al. Mental health outcomes of the CoViD-19 pandemic. Riv Psichiatr. 2020;55(3):137-44.

11. Choi KR, Heilemann M V, Fauer A, Mead M. A Second Pandemic: Mental Health Spillover From the Novel Coronavirus (COVID-19). Vol. 26, Journal of the American Psychiatric Nurses Association. United States; 2020. p. 340-3.

12. Safren SA, Blashill AJ, O'Cleirigh CM. Promoting the sexual health of MSM in the context of comorbid mental health problems. AIDS Behav. 2011 Apr;15 Suppl 1(0 1):S30-4.

13. Sanchez TH, Zlotorzynska $M$, Rai $M$, Baral SD. Characterizing the Impact of COVID-19 on Men Who
Have Sex with Men Across the United States in April, 2020. AIDS Behav [Internet]. 2020;(0123456789):19. Available from: http://link.springer.com/10.1007/ s10461-020-02894-2

14. Rearte $A$, Baldani $A$, Barcena Barbeira $P$, Domínguez C, Laurora $M$, Mena $M$, et al. Características epidemiológicas de los primeros 116974 casos de Covid-19 en Argentina, 2020. Rev Argentina Salud Pública. 2020;1-9.

15. BoletínOficialdelaRepúblicaArgentina. AISLAMIENTO SOCIAL PREVENTIVO Y OBLIGATORIO. 2020.

16. Ministerio de Salud de Argentina. 25-10-20-reportevespertino-covid-19. 2020;

17. Secretaria de Gobierno de Salud de la Nación. República Argentina 2019. Boletín sobre el VIH, sida e ITS en la Argentina. No 36 - AÑO XXII - DICIEMBRE DE 2019. 2019.

18. Ballivian J, Alcaide ML, Cecchini D, Jones DL, Abbamonte JM, Cassetti I. Impact of COVID-19related stress and lockdown on mental health among people living with HIV in Argentina. J Acquir Immune Defic Syndr. 2020 Sep;

19. Torres TS, Hoagland B, Bezerra DRB, Garner A, Jalil EM, Coelho LE, et al. Impact of COVID-19 Pandemic on Sexual Minority Populations in Brazil: An Analysis of Social/Racial Disparities in Maintaining Social Distancing and a Description of Sexual Behavior. AIDS Behav. 2020 Jul;1-12.

20. Stoloff K, Joska JA, Feast D, De Swardt G, Hugo J, Struthers $\mathrm{H}$, et al. A description of common mental disorders in men who have sex with men (MSM) referred for assessment and intervention at an MSM clinic in Cape Town, South Africa. AIDS Behav. 2013 May;17 Suppl 1:S77-81.

21. Sun S, Pachankis JE, Li X, Operario D. Addressing Minority Stress and Mental Health among Men Who Have Sex with Men (MSM) in China. Curr HIV/AIDS Rep. 2020 Feb;17(1):35-62.

22. White JM, Gordon JR, Mimiaga MJ. The Role of Substance Use and Mental Health Problems in Medication Adherence Among HIV-Infected MSM. LGBT Heal. 2014 Dec;1(4):319-22.

23. MACS/WIHS Combined Cohort Study. MWCCS COVID forms [Internet]. 2020. Available from: https://mwccs. org/covid-forms/

24. O’Cleirigh C, Magidson JF, Skeer MR, Mayer KH, Safren SA. Prevalence of Psychiatric and Substance Abuse Symptomatology Among HIV-Infected Gay and Bisexual Men in HIV Primary Care. Psychosomatics. 2015;56(5):470-8. 
25. Mann DM, Chen J, Chunara R, Testa PA, Nov O. COVID-19 transforms health care through telemedicine: Evidence from the field. J Am Med Inform Assoc. 2020 Jul;27(7):1132-5.

26. Ohannessian R, Duong TA, Odone A. Global Telemedicine Implementation and Integration Within Health Systems to Fight the COVID-19 Pandemic: A Call to Action. JMIR public Heal Surveill. 2020 Apr;6(2):e18810.

27. Hong Z, Li N, Li D, Li J, Li B, Xiong W, et al. Telemedicine During the COVID-19 Pandemic: Experiences From Western China. J Med Internet Res. 2020 May;22(5):e19577.

28. Niles MT, Bertmann F, Belarmino EH, Wentworth $T$, Biehl E, Neff R. The Early Food Insecurity Impacts of COVID-19. Nutrients. 2020 Jul;12(7). 
Impact of COVID-19 pandemic and lockdown among Men who have sex with Men living with HIV in Argentina

Background: People living with HIV (PLWH) are at increased risk of mental health complications. In order to mitigate the spread of COVID-19 in our country, an early quarantine was established. We studied the impact of COVID-19-related stress and quarantine in men who have sex with men living with HIV(MSMLH) in Argentina.

Materials and methods: An anonymous survey was conducted between April and May 2020. PLWH in followup at a private institution in Argentina were included. We assessed adherence to preventive measures of COVID-19 and antiretroviral treatment, access to resources, psychosocial factors and substance use during quarantine. This analysis focused on MSMLH results.

Results: Among 1336 participants included, 596 self-reported as MSMLH (median age 44, IQR 35-52). Quarantine adherence was $96.8 \%$; household job loss $55.4 \%$. Interruption to access to antiretroviral medication was observed in $4.9 \%$ and $29.9 \%$ reported suboptimal adherence. Mental health problems were common: depression (14.1\%), loneliness (15.6\%) anxiety (20.2\%).

Conclusions: Our study showed high adherence to preventive measures during the beginning of lockdown and difficulties to access health services. The COVID-19 pandemic has compromised the country's economy; this study shows its impact on MSMLH access to basic resources. Since MSMLH mental health has been affected, the pandemic highlights the need of a comprehensive approach to our patients' health.

Keywords: HIV; COVID-19; Argentina; mental health. 